\title{
Heterogeneity in myocardial iron content in pediatric and young adult patients
}

\author{
Tim Slesnick, Jeanne Boudreaux, Denver Sallee, WJ Parks \\ From 16th Annual SCMR Scientific Sessions \\ San Francisco, CA, USA. 31 January - 3 February 2013
}

\begin{abstract}
Background
Non-invasive quantification of myocardial iron content in patients with chronic anemia by Cardiac Magnetic Resonance (CMR) using T2* imaging sequences has significantly improved their clinical care. Measurement of myocardial iron content is traditionally performed on the interventricular septum of a single, mid-ventricular short axis image. Limited data exists to evaluate the reproducibility of myocardial $\mathrm{T} 2 *$ imaging in pediatric and young adult patients. Furthermore, there is no data in this patient group evaluating the heterogeneity of myocardial iron throughout the ventricular septum (apex versus mid versus basilar levels).
\end{abstract}

\section{Methods}

All patients underwent standardized T2* imaging with a multiecho, gradient echo sequence obtained during a single breath hold, with 3 copies of the mid-ventricular slice repeated. The protocol was then expanded to include apical, mid, and basilar level slices, with repeated acquisitions at each location. Retrospective review with re-measurement of all T2* values was performed using a local workstation.

\section{Results}

Ten patients had repeated measurements of the their midventricular T2* imaging, and an additional 8 patients had measurements at three slice locations (9/18 males, median age 19.3 years, range 11.2-23.6 years). For the initial 10 patients, the median $\mathrm{T} 2 *$ value was $21.3 \mathrm{~ms}$ (range 6.336.0 ), with a median standard deviation of $1.5 \mathrm{~ms}$ (range 0.1-4.4) at the mid-ventricular level. Two of ten patients had values that spanned the clinically accepted cut-point of $20 \mathrm{~ms}$, indicating they could have been classified as either normal or mildly abnormal depending on which image was selected for analysis. For the 8 patients with multiple slice locations analyzed, the median $\mathrm{T} 2 *$ values were $29.5 \mathrm{~ms}$ (9.5-47.2), 25.9 (12.5-37.3), and 22.7 (9.933.7) for the apex, mid, and basilar levels respectively. While the median standard deviations for each slice location were low $(0.6,0.9$, and 0.5 respectively), the differences between the slice locations resulted in 1 patient having values both above and below $20 \mathrm{~ms}$, and 2 additional patients with values above and below the "severely abnormal" cut-point of $10 \mathrm{~ms}$.

\section{Conclusions}

Variability exists in $\mathrm{T} 2 *$ values for both same slice position and different slice positions from apex to basilar interventricular septal myocardial segments in pediatric and young adult patients. Five of 18 patients had values both above and below clinically accepted cut-points for normal, mildly abnormal, and severely abnormal iron content on separate images. This heterogeneity in myocardial iron content implies that a more comprehensive routine myocardial evaluation may be indicated for these patients.

\section{Funding}

None.

Published: 30 January 2013

doi:10.1186/1532-429X-15-S1-P287

Cite this article as: Slesnick et al:: Heterogeneity in myocardial iron content in pediatric and young adult patients. Journal of Cardiovascular Magnetic Resonance 2013 15(Suppl 1):P287.

Pediatrics, Emory University, Atlanta, GA, USA

(c) 2013 Slesnick et al; licensee BioMed Central Ltd. This is an Open Access article distributed under the terms of the Creative Commons Attribution License (http://creativecommons.org/licenses/by/2.0), which permits unrestricted use, distribution, and reproduction in any medium, provided the original work is properly cited. 